Research Article

\title{
Determination of genetic variation in Rhodiola crenulata from the Hengduan Mountains Region, China using inter-simple sequence repeats
}

\author{
Yidong Lei ${ }^{1,2}$, Hong $\mathrm{Gao}^{2}$, Tashi Tsering ${ }^{3}$, Suhua Shi ${ }^{4}$ and Yang Zhong ${ }^{2}$ \\ ${ }^{1}$ Fudan University, Department of Environmental Science and Engineering, Shanghai, China. \\ ${ }^{2}$ Ministry of Education, Fudan University, School of Life Sciences, Key Laboratory for Biodiversity Science \\ and Ecological Engineering, Shanghai, China. \\ ${ }^{3}$ Tibet University, Department of Biology, Lhasa, Tibet, China. \\ ${ }^{4}$ Ministry of Education, Zhongshan University, Key Laboratory of Gene Engineering, Guangzhou, China.
}

\begin{abstract}
The plant Rhodiola crenulata is a perennial herbaceous species distributed in the plateau region of southwestern China, especially the Hengduan Mountains region. It has been one of the most important traditional herbal remedies in Tibet for more than one thousand years, but the accelerated and uncontrolled collection of this plant since the 1980 s has lead to deforestation. We used inter-simple sequence repeats (ISSR) to assess levels of genetic variation in $R$. crenulata from nine diverse natural populations in eastern Tibet and northern Yunnan, the first time such a study has been carried out. The 12 primers we used were able to detect 184 polymorphic loc. Analysis of molecular variance (AMOVA) indicated that species level genetic diversity was relatively high $\left(p=97.83 \%\right.$, and $\left.H_{0}=0.464\right)$ and analysis using Shannon's index showed that the within and between genetic diversity of $R$. crenulata are approximately equal. Nei's genetic distance and unweighted pair-group method with arithmetic averages (UPGMA) cluster analysis showed that the three populations from Tibet and the six populations from Yunnan form two major clusters. The Yunnan populations from three locations were further divided into three corresponding groups, indicating that genetic differentiation was correlated to geographic distribution. Understanding the genetic structure of $R$. crenulata provides insight for the conservation and management of this endangered species.
\end{abstract}

Key words: genetic diversity, Hengduan Mountains region, ISSR, Rhodiola crenulata.

Received: January 1, 2005; Accepted: November 16, 2005.

\section{Introduction}

The plant Rhodiola crenulata is a perennial herbaceous plant mainly distributed in the high plateau region of southwestern China, especially the Hengduan Mountains region including eastern Tibet, northern Yunnan and western Sichuan. Thriving at high elevations (2800-5600 m) in cold, moist climatic areas of the Northern hemisphere near the tundra, $R$. crenulata grows exceptionally well in a variety of habitats including meadows, rocky crevices, cliffs and slopes, dunes and sandy soils. A cluster of $R$. crenulata plants often grows to several tens of square meters and produces reptant, succulent rhizomes and red extracting solutions from flowers, roots and stems ( $\mathrm{Fu}$ and $\mathrm{Fu}, 1984 ; \mathrm{Wu}$ and Raven, 2001). As one of the most important traditional herbal remedies, $R$. crenulata has long been used in the

Send correspondence to: Yang Zhong. Ministry of Education, Fudan University, School of Life Sciences, Key Laboratory for Biodiversity Science and Ecological Engineering, 200433 Shanghai, China. E-mail: yangzhong@fudan.edu.cn. treatment of long-term illnesses and weaknesses due to infection in Tibet and other regions for more than 1000 years (Xiong, 1995; Zhao et al., 1998; Rohloff, 2002). Recent pharmacological studies have found that this plant contains many ingredients such as salidrosides which are highly active against anoxia, fatigue, toxic reactions, radiation sickness, tumors and aging as well as presenting active-oxygen scavenging properties (Kurkin and Zapesochnaya, 1986; Peng et al., 1996; Ohsugi et al., 1999). Lei et al. (2003, 2004) investigated the interpopulation chemical variability of essential oils from $R$. crenulata rhizomes from Tibet and Yunnan and found that there were two main chemotypes of the essential oils in Tibetan (geraniol-rich) and Yunnan (n-octanol- and geraniol-rich) $R$. crenulata populations which can be used for identifying the source of $R$. crenulata genetic resources.

Since 1980s, the accelerated and uncontrolled use of $R$. crenulata in southwestern China has been leading to deforestation, with a number of Rhodiola species, including $R$. crenulata, considered for inclusion in the National Class 
One Endangered Species in China checklist for conservation purpose. Little is known about the genetic background of $R$. crenulata and there is a need for more information on genetic variability and the population structure of natural populations to support $R$. crenulata conservation and management programs.

In recent years a number of molecular markers such as random amplified polymorphic DNA (RAPD) (Hu and Quiros, 1991; Munthali et al., 1992), amplified fragment length polymorphism (AFLP) (Vos et al., 1995), simple sequence repeats (SSR) (Zietkiewicz et al., 1994) and intersimple sequence repeats (ISSR) (Salimath et al., 1995; Wolfe and Randle, 2001) have been widely used to detect genetic diversity in plants (Karp et al., 1996; Wolfe and Liston, 1998; Nan et al., 2003; Tang et al., 2003). In particular, ISSR markers can be highly variable within a species and have the advantage over RAPD markers that they use longer primers that allow more stringent annealing temperatures and reveal many more polymorphic fragments. In this study, ISSR markers were used to analyze the genetic diversity and genetic structure of natural $R$. crenulata populations from the Hengduan Mountains region, China. The objectives of the research described in this paper was to evaluate the population-level genetic diversity of $R$. crenulata for the first time, to determine the genetic differentiation between the populations from Tibet and Yunnan, China, and to assess the implications of our study for future Rhodiola conservation and management programs.

\section{Materials and Methods}

\section{Sample collection}

A total of 164 Rhodiola crenulata plants, representing nine diverse natural populations, were sampled from the Hengduan Mountains region, China (Figure 1). Fresh $R$. crenulata leaves were collected from the following four sets of sites: (1) Kongpojiangda (Kongpo Gyamda) County, Linzhi (Nyingchi) District, Tibet in August 2002 from Mount Mila, eastern side at $4670 \mathrm{~m}$ (sample P1), western side at $5150 \mathrm{~m}$ (sample P2) and western side at 3890 m (sample P3); (2) Deqin County, Diqing District, Yunnan, China in September 2002 from Mount Meili Snow at $4650 \mathrm{~m}$ (sample P4); (3) the mining region, Shangri-la County, Diqing District, Yunnan in August 2003 from Mt. Hong at $4390 \mathrm{~m}$ (sample P5), $4420 \mathrm{~m}$ (sample P6) and $4420 \mathrm{~m}$ (P7); and (4) Lijiang, Yunnan in July, 2003 from Mount Jade Dragon Snow at $5100 \mathrm{~m}$ (P8) and $4100 \mathrm{~m}$ (P9). The leaves were collected from 12 - 23 randomly selected plants in each site (population) at intervals of at least $4 \mathrm{~m}$. Samples were stored with silica gel in zip-lock plastic bags until DNA isolation. Vouchers of the samples were deposited at the Ministry of Education Key Laboratory for Biodiversity Science and Ecological Engineering at Fudan University, Shanghai 200433, China.
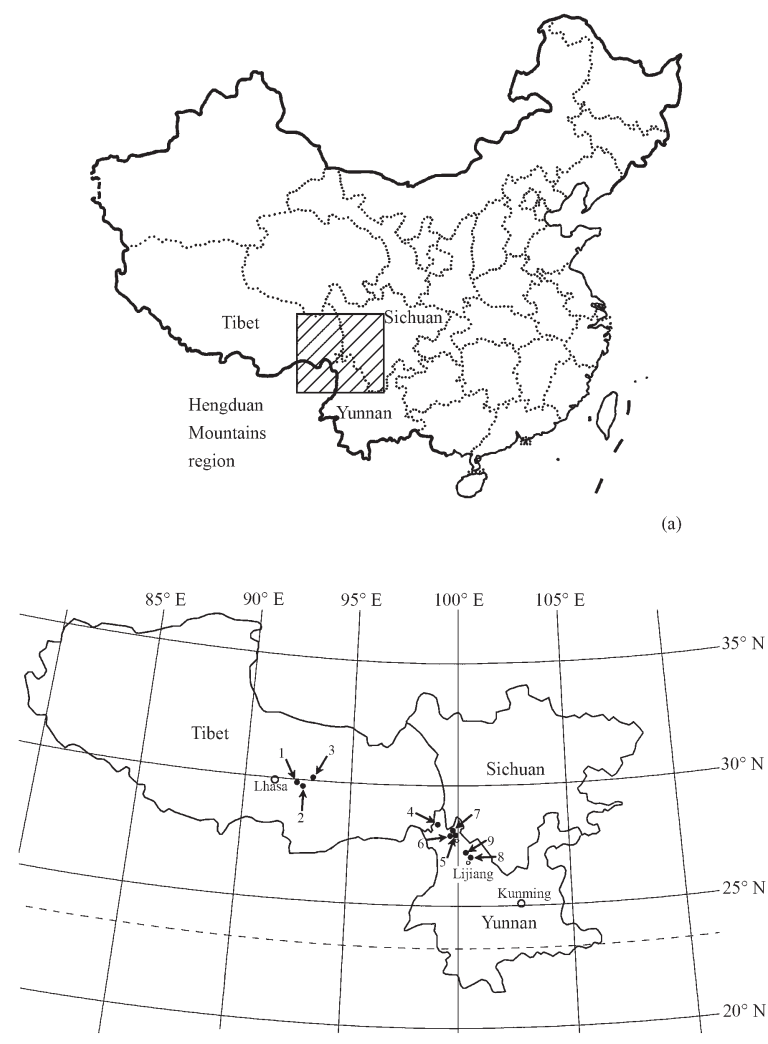

(b)

Figure 1 - Samples of nine Rhodiola crenulata populations from the Hengduan Mountains region, China. (a) the Hengduan Mountains region; (b) sampling locations. Population codes and the number of plants sampled are given in Table 2.

\section{DNA extraction and ISSR assay}

A modified DNA mini-prep procedure of Doyle and Doyle (1990) was used to extract DNA, the quality of which was checked on $1 \%(\mathrm{w} / \mathrm{v})$ agarose gel and its concentration determined using a Hoeffer fluorometer.

Ninety primers from the University of British Columbia, Canada set 9 of ISSR primers (http://www. michaelsmith.ubc.ca) were tested and 12 primers were selected for PCR (Table 1). Reaction volumes were $10 \mu \mathrm{L}$, and consisted of $1 \mu \mathrm{L} 10 \times$ reaction buffer, $0.1 \mu \mathrm{L} 10 \mathrm{mM}$ dNTPs, $0.25 \mu \mathrm{M}$ primer, $2 \%$ (w/v) formamide, $10 \mathrm{ng}$ DNA template and 1 unit of Taq DNA polymerase (Genda Tech Corp, Canada). The thermocycler program was $5 \mathrm{~min}$ at $94{ }^{\circ} \mathrm{C}$ followed by 40 cycles of $1 \mathrm{~min}$ at $94{ }^{\circ} \mathrm{C}, 1 \mathrm{~min}$ annealing at 51 or $54.5^{\circ} \mathrm{C}$ (Table 1) and 2 min extension at $72{ }^{\circ} \mathrm{C}$, and a final $7 \mathrm{~min}$ extension at $72{ }^{\circ} \mathrm{C}$.

Amplification products were resolved electrophoretically on $1.5 \%(\mathrm{w} / \mathrm{v})$ agrose gels in $0.5 \mathrm{x}$ TBE buffer by loading the entire reaction volumes into prepared wells. Gels were run until a bromophenol blue indicator dye ran $10 \mathrm{~cm}$ from the well. Gels were stained with ethidium bromide and bands were visualized and photographed under 
Table 1 - Primers used for ISSR amplification.

\begin{tabular}{llc}
\hline Primer & Sequence & Annealing temperature $\left({ }^{\circ} \mathrm{C}\right)$ \\
\hline 807 & $(\mathrm{AG})_{8} \mathrm{~T}$ & 53 \\
810 & $(\mathrm{GA})_{8} \mathrm{~T}$ & 52 \\
811 & $(\mathrm{GA})_{8} \mathrm{C}$ & 52 \\
823 & $(\mathrm{TC})_{8} \mathrm{C}$ & 52 \\
834 & $(\mathrm{AG})_{8}(\mathrm{CT}) \mathrm{T}$ & 54.5 \\
840 & $(\mathrm{GA})_{8}(\mathrm{CT}) \mathrm{T}$ & 53 \\
841 & $(\mathrm{GA})_{8}(\mathrm{CT}) \mathrm{C}$ & 53 \\
845 & $(\mathrm{CT})_{8}(\mathrm{AG}) \mathrm{G}$ & 52 \\
857 & $(\mathrm{AC})_{8}(\mathrm{CT}) \mathrm{G}$ & 53 \\
864 & $(\mathrm{ATG})_{6}$ & 52 \\
873 & $(\mathrm{GACA})_{4}$ & 51 \\
887 & $(\mathrm{AGT})(\mathrm{ACG})(\mathrm{AGT})(\mathrm{TC})_{7}$ & 51 \\
\hline
\end{tabular}

UV light. Molecular weights were estimated using a $100 \mathrm{bp}$ DNA ladder (Shengong Inc., Shanghai, China).

\section{Scoring and data analysis}

All ISSR bands were scored as present (1) or absent (0). From banding patterns of the nine populations, the number of polymorphic loci and the percentage of polymorphic loci ( $p$ ) at population and species level were calculated. The degree of within-population diversity was quantified using Shannon's index of phenotypic diversity $\left(H_{o}\right)$, estimated as $-\Sigma p 3 \log _{2} p i$, where $p i$ was the frequency of the presence or absence of a band (Lewontin 1972; King and Schaal 1989). The average diversity over the different populations $\left(H_{p o p}\right)$ and the diversity calculated from the phenotypic frequencies $p$ in all populations considered together $\left(H_{s p}\right)$ was calculated from $-\Sigma p \log _{2} p$. It was then possible to calculate the proportion of diversity within $\left(H_{p o p}\right.$ $\left./ H_{s p}\right)$ and between populations $\left[\left(H_{s p}-H_{p o p}\right) / H_{s p}\right]$.

A dendrogram based on Nei's (1978) unbiased genetic distances and the unweighted pair-group method with arithmetic averages (UPGMA) was constructed using the
TFPGA program version 1.3 (Miller, 1997). Marker frequencies were estimated based on Lynch and Milligan's (1994) frequency correction for dominant markers. Bootstrapping over loci was also performed with TFPGA with 1000 permutations.

The correlation between the genetic distance and geographic distance matrices was investigated using the Mantel test of matrix correspondence (Mantel, 1967) implemented in the NTSYSpc 2.0 package (Rohlf, 1998). Statistical significance of the Mantel test was determined using 1000 random permutations.

Analyses of molecular variance (AMOVA) based on the pairwise squared Euclidean distances between molecular phenotypes were carried out to partition the genetic diversity between populations using the WINAMOVA program version 1.55 (Excoffier et al., 1992). Gene flow as number of migrants per generation $\left(N_{m}\right)$ between populations was estimated based upon $\Phi_{\mathrm{ST}}$ (analogous to $F_{\mathrm{ST}}$ ), using the method of Wright (1951).

\section{Results}

For the $164 R$. crenulata specimens tested the 12 primers produced 184 bands, 180 of which were polymorphic. The size of the amplified DNA fragments ranged from $190 \mathrm{bp}$ to $2600 \mathrm{bp}$ and the average number of bands per primer was 15.3. The number of bands and polymorphic bands produced by each primer varied, the highest number of bands (20) being produced by primer 845 and the lowest number of bands (11) by primer 807 . We observed 164 unique ISSR banding patterns, i.e., each specimen presented a unique ISSR phenotype, indicating extensive genetic variation in the individual plants analyzed. There were no population-specific markers, i.e. markers present in one population but absent in the others.

The percentage of polymorphic loci ranged from $38.04 \%$ for population P3 to $66.85 \%$ for population $\mathrm{P} 7$, with a mean of about $56.28 \%$ (Table 2 ). The percentage of polymorphic bands at species level for each primer combinations ranged from about $92.31 \%$ to $100 \%$. Estimates of

Table 2 - Genetic variation between populations of Rhodiola crenulata based on 184 ISSR markers.

\begin{tabular}{|c|c|c|c|c|}
\hline Population & Sample size & Total number of polymorphic loci & Percentage of polymorphic loci & $H_{o}($ mean $\pm \mathrm{SE})$ \\
\hline P1 & 11 & 92 & 50.00 & $0.254 \pm 0.286$ \\
\hline P2 & 15 & 104 & 56.52 & $0.282 \pm 0.284$ \\
\hline P3 & 21 & 70 & 38.04 & $0.168 \pm 0.245$ \\
\hline P4 & 12 & 111 & 60.33 & $0.291 \pm 0.272$ \\
\hline P5 & 20 & 111 & 60.33 & $0.279 \pm 0.268$ \\
\hline P6 & 20 & 100 & 54.35 & $0.247 \pm 0.270$ \\
\hline P7 & 23 & 123 & 66.85 & $0.325 \pm 0.275$ \\
\hline P8 & 20 & 113 & 61.41 & $0.274 \pm 0.267$ \\
\hline P9 & 22 & 108 & 58.70 & $0.295 \pm 0.282$ \\
\hline Total & 164 & 180 & 97.83 & $0.464 \pm 0.204$ \\
\hline
\end{tabular}


Shannon's index of phenotypic diversity $\left(H_{o}\right)$ ranged from 0.168 for population $\mathrm{P} 3$ to 0.325 for population P7 with an average of 0.268 , which was consistent with the variance of the percentage of polymorphic bands. At species level, $H_{o}$ was 0.464 (Table 2).

Partitioning of genetic variability by analysis of molecular variance revealed that most of the ISSR diversity was distributed between individual plants within populations $(52.62 \%)$, with the remaining diversity distributed between populations within locations $(25.36 \%)$ and between locations (22.02\%) (Table 3). Of the total genetic diversity, $44.86 \%$ resided in populations and the rest $(55.14 \%)$ in individual plants within populations. We also found that $32.92 \%$ of the variance was between locations and $67.08 \%$ within locations. The estimated population differentiation $\left(\Phi_{\mathrm{ST}}=0.474\right)$ for this phenetic treatment of the data was close to the coefficient of Shannon's index. Based on the $\Phi_{\text {ST }}$ value, the gene flow $\left(N_{m}\right)$ was 0.277 .

A dendrogram generated using the Nei's (1978) genetic distance and the UPGMA method showed that the Tibetan populations and Yunnan populations form two major clusters, implying that the genetic differentiation of $R$. crenulata is correlated to its geographic distribution. The Yunnan populations were divided into three groups (Figure 2): population P4 from Meili snow mountain; three closely related populations from Mt. Hong (P5, P6, and P7); and populations P8 and P9, which were also similar to each other, from Lijiang (Jade Dragon Snow Mountain).

The Mantel test revealed that there was significant correlation between pairwise genetic distance and corresponding geographic distance for all nine populations $(r=0.677, \mathrm{p}=0.0060)$, consistent with the UPGMA dendrogram that clusters closely-related populations.

\section{Discussion}

This study is the first application of ISSR markers to the assessment of genetic diversity in $R$. crenulata. The partition of genetic diversity using both Shannon's index and AMOVA indicated that genetic diversity in $R$. crenulata is distributed equally within and between populations. Since the population genetic structure of a species is affected by multiple evolutionary factors including the mating system,

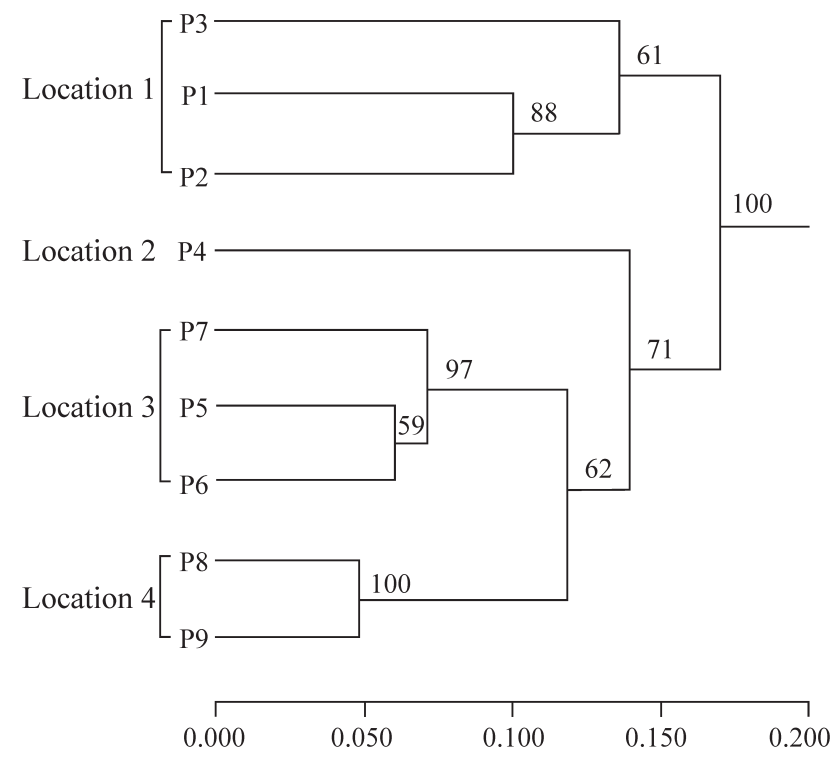

Figure 2 - Dendrogram showing the relationships between nine Rhodiola crenulata populations. The dendrogram was generated using the unweighted pair-group method with arithmetic averages (UPGMA) method using the genetic distances matrix based on 184 inter-simple sequence repeat (ISSR) markers produced by 12 single primers. Bootstrap support values are indicated above the branches.

gene flow, mode of reproduction and natural selection (Hamrick and Godt, 1989), it could be speculated that $R$. crenulata might have a mixed mating system, partial outcrossing by pollen and seed dispersal and partial selfing by sprouting prostrate rhizomes. In general, the detection of high levels of polymorphism makes ISSR analysis a powerful tool for assessing genetic diversity in $R$. crenulata. None of the individual plants were genetically identical according to ISSR analysis, indicating that the level of resolution in our study was sufficient to distinguish all genotypes.

Accurate estimates of genetic diversity are useful for optimizing sampling strategies and for conserving and managing the genetic diversity of trees (Godt and Hamrick, 1996). In the present study, the genetic diversity indices $\left(\mathrm{p}=97.83 \%, H_{o}=0.464\right)$ revealed that genetic diversity of $R$. crenulata from the Hengduan Mountains region in southwestern China is relatively high in comparison to that recorded for Rhodiola angusta $(\mathrm{p}=46.7 \%, \mathrm{H}=0.195)$ and Rhodiola sachalinensis ( $\mathrm{p}=30.7 \%, H=0.122$ ) based on

Table 3 - Analysis of Molecular Variance (AMOVA) of Rhodiola crenulata based on 184 inter-simple sequence repeat (ISSR) markers.

\begin{tabular}{|c|c|c|c|c|c|c|c|}
\hline \multirow{3}{*}{$\begin{array}{l}\text { Source of } \\
\text { variation }\end{array}$} & \multirow{3}{*}{$\begin{array}{c}\begin{array}{c}\text { Degrees of } \\
\text { freedom }\end{array} \\
3\end{array}$} & \multirow{3}{*}{$\begin{array}{c}\begin{array}{c}\text { Sum of squared } \\
\text { deviation }\end{array} \\
1339.1320\end{array}$} & \multirow{3}{*}{$\begin{array}{c}\begin{array}{c}\text { Mean of squared } \\
\text { deviation }\end{array} \\
446.377\end{array}$} & \multirow{2}{*}{\multicolumn{2}{|c|}{$\begin{array}{c}\text { Variance component } \\
\text { Absolute }(\%)\end{array}$}} & \multirow{3}{*}{$\begin{array}{c}\text { Coefficient of } \\
\text { intraclass correlation } \\
\Phi_{\mathrm{ct}}=0.220\end{array}$} & \multirow{3}{*}{$\begin{array}{c}\mathrm{p}^{*} \\
<0.001\end{array}$} \\
\hline & & & & & & & \\
\hline & & & & 7.38 & 22.02 & & \\
\hline Between populations & 5 & 879.7345 & 175.947 & 8.50 & 25.36 & $\Phi_{\mathrm{sc}}=0.325$ & $<0.001$ \\
\hline Within populations & 155 & 2733.5420 & 17.63 & 17.64 & 52.62 & $\Phi_{\mathrm{st}}=0.474$ & $<0.001$ \\
\hline Total & 163 & 4952.4085 & 30.383 & 33.52 & 100 & & \\
\hline
\end{tabular}

*Based on 1000 iteration steps. 
isozyme markers (Yan et al., 1999; Zu et al., 1998). The $R$. crenulata genetic diversity detected in our study also agrees with the life history traits and geographical distribution of this species. Although the genus Rhodiola originates in the mountainous regions of Southwest China and the Himalayas, botanists have established that various species of this genus naturally display a circumpolar distribution in the mountainous regions at the very high latitudes and elevations of the Northern Hemisphere (Ohba, 1987; Darbinyan et al., 2000).

It is known that $R$. crenulata reproduces both sexually and vegetatively by sprouting prostrate rhizomes, and this can be considered as a strategy for both maximal heterozygosity and reproductive success. The high genetic diversity observed in our investigation indicates that the populations studied have adapted to environmental changes. One of the reasons is that the locations of the sample collection were in the Hengduan Mountains region, which is one of the biodiversity hotspots of the world and the current distribution and differentiation center for the genus Rhodiola. However, the fact that Rhodiola natural resources have decreased remarkably recently, owning to over-exploitation for medicine and shrinkage of their natural habitat, indicates that the threats to the survival of species of this genera mainly come from human activity and not genetic variation.

Andrew (1999) states that understanding the evolutionary role of gene flow or the migration of individual specimens and the subsequent transfer of genes between populations is also pivotal to the management of endangered species. The indirect estimate of gene flow $(\mathrm{Nm})$ between $R$. crenulata populations was extremely low (0.277), implying that genetic drift could be the dominant evolutionary factor that shapes the population structure of $R$. crenulata according to Wright (1931). Moderate to high rates of gene flow between populations help prevent subpopulation isolation and thereby maintain genetic variation and prevent inbreeding depression (Franklin, 1980; Frankel and SouleÄ, 1981). Thus with a very low migration rate, genetic drift could have effectively isolated and differentiated the $R$. crenulata populations after a long period, consistent with the almost equal percentage of within and between populations genetic variation. Another potential factor affecting the loss of genetic variation is habitat fragmentation, which could have restricted gene flow between $R$. crenulata populations as evidenced by the fact that this species could only be found in the high and cold mountainous regions.

The $R$. crenulata population structure revealed by our research has apparent conservation and management implications. An important goal for the conservation of this species is to preserve more individual plants than populations. Accordingly, in a situation of limited collection and management capacity in germplasm conservation, our recommendation is to collect more individual plants from each population but fewer populations. Although loss of individual plants or populations in some locations may not cause the immediate loss of genetic diversity, it might result in the decreased potential of $R$. crenulata to adapt to environmental changes in the long term. If the goal of rehabilitation and restoration is functionally equivalent with native local populations, the donor propagules should be selected from populations that have high genetic similarity to one another. For example, in planting $R$. crenulata in China, propagules collected from ecologically similar populations should be used to increase their potential for survival. In contrast, if the sites are to be planted with propagules from donor populations, then sampling strategies should focus on small collections spreading across populations and covering several sites on a regional scale to obtain the maximum representation of genetic diversity.

\section{Acknowledgments}

We thank Mr. Shuangquan Duan (Tibet University, TU) and Zhankui Bai (Fudan University, FU) for their help in fieldwork, Miss FengxiaoTan (Zhongshan University, $\mathrm{ZU}$ ) and Li Wang (FU) for ISSR technical assistance, and Miss TianTang (ZU) for data analysis and critical comments on previous versions of this paper. This research was partially supported by the National Natural Science Foundation of China (grant numbers 30360011, 30230030) and the Chinese National Key Project for Basic Research (973) (grant number 2002 CB 512801).

\section{References}

Andrew S (1999) Gene flow and endangered species translocations: A topic revisited. Biolo Conserv 87:173-180.

Darbinyan V, Kteyan A, Panossian A, Gabrielian E, Wikman G and Wagner H (2000) Rhodiola rosea in stress induced fatigue: A double blind cross-over study of a standardized extract SHR-5 with a repeated low-dose regimen on the mental performance of healthy physicians during night duty. Phytomedicine 7:365-71.

Doyle JJ and Doyle JL (1990) Isolation of plant DNA from fresh tissue. Focus 12:13-15.

Excoffier L, Smouse PE and Quattro JM (1992) Analysis of molecular variance inferred from metric distances among DNA haplotypes: Application to human mitochondria DNA restriction sites. Genetics 131:479-491.

Frankel OH and SouleÄ ME (1981) Conservation and Evolution. Cambridge University Press, UK, 327 pp.

Franklin IR (1980) Evolutionary change in small populations. In: SouleÂ ME and Wilcox BA (eds) Conservation Biology: An Evolutionary-Ecological Perspective. Sinauer Associates, Massachusetts, pp 135-150.

Fu SX and Fu KJ (eds) (1984) Flora Republicae Popularis Sinicae. Science Press, Beijing, v. 34, pp 159-220.

Godt MJW and Hamrick JL (1996) Genetic diversity and morphological differentiation in Liatris helleri (Asteraceae), a threatened plant species. Biodiv Conserv 5:461-471

Hamrick JL and Godt MJW (1989) Allozyme diversity in plant species. In: Brown AHD, Clegg MT, Kahler Al and Wier BS 
(eds) Plant Population Genetics, Breeding, and Genetic Resources. Sunderland, MA, Sinauer Associates, pp 43-63.

$\mathrm{Hu}$ J and Quiros CF (1991) Identification of broccoli and cauliflower cultivars with RAPD markers. Pl Cell Rep 10:505511.

Karp A, Seberg O and Bulatti M (1996) Molecular techniques in the assessment of botanical diversity. Ann Bot 78:143-149.

King L M and Schaal BA (1989) Ribosomal DNA variation and distribution in Rudbeckia missouriensis. Evolution 43:1117-1119.

Kurkin VA and Zapesochnaya GG (1986) Chemical composition and pharmacological studies of Rhodiola plants. Khim Farm Zh 20:231-1244 (in Russian).

Lei YD, Nan P, Tsering T, Bai ZK, Tian CJ and Zhong Y(2003) Chemical composition of the essential oils of two Rhodiola species from Tibet. Z Naturforsch 58c:161-164.

Lei YD, Nan P, Tsering T, Wang L, Liu SP and Zhong Y(2004) Interpopulation variability of rhizome essential oils in Rhodiola crenulata from Tibet and Yunnan, China. Biochem Syst Ecolo 32:611-614.

Lewontin RC (1972) The apportionment of human diversity. Evol Biol 6:381-398.

Lynch M and Milligan BG (1994) Analysis of population genetic structure with RAPD markers. Mol Ecol 3:91-99.

Mantel NA (1967) The detection of disease clustering and a generalized regression approach. Cancer Res 27:209-220.

Miller MP (1997) Tools for Population Genetic Analyses (TFPGA), Version 1.3. Department of Biological Sciences, Northern Arizona University, Flagstaff, AZ.

Munthali M, Ford-Lloyd BV and Newbury HJ (1992) The random amplification of polymorphic DNA for fingerprinting plants. PCR Methods Appl 1:274-276.

Nan P, Shi SH, Peng SL, Tian CJ and Zhong Y (2003) Genetic diversity in Primula obconica (Primulaceae) from central and south-west China as revealed by ISSR markers. Ann Bot 91:329-333.

Nei M (1978) Estimation of average heterozygosity and genetics distance from a small number of individuals. Genetics 89:583-590.

Ohba H (1987) Biogeography of genus Rhodiola (Crassulaceae). Acta Phytotax Geobot 38:211-223.

Ohsugi M, Fan W, Hase K, Xiong Q, Tezuka Y, Komatsu K, Namba T, Saitoh T, Tazawa K and Kadota S (1999) Active-oxygen scavenging activity of traditional nourishingtonic herbal medicines and active constituents of Rhodiola sacra. J Ethnopharmacol 67:111-119.
Peng JN, Ge YC and Li XH (1996) Studies on the chemical constituents of Rhodiola fastigita. Acta Pharm Sin 31:798-800.

Rohlf FJ (1998) NTSYSpc: Numerical Taxonomy and Multivariate Analysis System, version 2.02. Exeter Software, Setauket, NY.

Rohloff J (2002) Volatiles from rhizomes of Rhodiola rosea L. Phytochemistry 59:655-661.

Salimath SS, De Oliverira AC, Godwin ID and Bennetzen JL (1995) Assessment of genomic origins and genetic diversity in the genus Eleusine with DNA markers. Genome 38:757763.

Tang T, Zhong Y, Jian SG and Shi SH (2003) Genetic diversity of Hibiscus tiliaceus (Malvaceae) in China assessed using AFLP markers. Ann Bot 92:409-414.

Vos P, Hogers R, Bleeker M, Reijans M, vander der Lee T, Hornes M, Frijters A, Pot J, Wheeler T and Peleman J (1995) AFLP: A new technique for DNA fingerprinting. Nucl Acid Res 23:4407-4414.

Wolfe AD and Liston A (1998) Contributions of PCR-based methods to plant systematics and evolutionary biology. In: Soltis PS, Soltis DE and Doyle JJ (eds) Molecular Systematics of Plant: DNA Sequencing. Kluwer, New York, pp 43-86.

Wolfe AD and Randle CP (2001) Relationships within and among species of the holoparasitic genus Hyobanche (Orobanchaceae) inferred from ISSR banding patterns and nucleotide sequences. Syst Bot 26:120-130.

Wright S (1931) Evolution in Mendelian populations. Genetics 16:97-159.

Wright S (1951) The genetical structure of populations. Ann Engen 15:323-354.

Wu Z and Raven PH (eds) (2001) Flora of China. Science Press, Beijing, and St. Louis. Missouri Botanical Garden Press, v 8, pp 251-268.

Xiong RX (1995) An investigation of the resources of Rhizoma rhodiolae in Tibet. WCJ PS 10:187-188.

Yan TF, Zhou FJ, Yan XF and Zu YG (1999) Genetic diversity and population differentiation of Rhodiola angusta. Bull Bot Res 19:189-194.

Zhao YH, Liu CH and Wu TH (1998) Researches and uses of Rhodiola plants. Forest By-Prod. Special. China 3:44-45.

Zietkiewicz E, Rafalski A and Labuda D (1994) Genome fingerprinting by simple sequence repeats (SSR)-anchored PCR amplification. Genomics 20:176-183.

Zu YG, Yan TF and Zhou FJ (1998) A preliminary study on genetic variation and endangered mechanism of Rhodiola sachalinensis natural population. Bull Bot Res 18:304-310. Associate Editor: Everaldo Gonçalves Barros 